\title{
Navigability Testing for Web Applications - A Tool based Approach
}

\author{
R. Jayakarthik \\ Research Scholar \\ Madurai Kamaraj University, \\ Madurai, India.
}

\author{
K. Alagarsamy \\ Associate Professor \\ Madurai Kamaraj University \\ Madurai, India
}

\begin{abstract}
Web Engineering - the branch of Software Engineering dealing with the development and maintenance of quality web sites has become the apple of the eye for many researchers in the domain of Software Engineering. This can be attributed to the proliferation in the number of web sites being developed and their strategic impact on various business domains. NonFunctional Requirements (NFRs) occupy a primal position in Web Engineering as they do in traditional Software Engineering. This paper presents the results of a case study on Load and Performance Testing applied to a Web Application and proposes a new tool for Navigability Testing
\end{abstract}

\section{Keywords}

Navigability, Testing, Web Applications

\section{INTRODUCTION}

Owing to the increasing inclination of people towards the World Wide Web, Software Engineering researchers have been studying the unique aspects of Web Engineering for the past few years. There has been a steep increase in the number of web sites being engineered and web sites have also become vital to the success of business organizations across multiple domains.

Non-Functional Requirements for conventional non-web based applications like user-friendliness, adaptability are being researched extensively. It turns out that these NFRs are also extremely important in Web Engineering. Infact, the authors of the paper feel they are more important in Web Engineering than in the Engineering of Non-Web based applications.

The paramount importance of NFRs in Web Engineering has necessitated testing strategies devoted to the testing of NFRs in Web Engineering.

The 2 main categories of NFRs to be tested for web sites are Load \& Performance and Navigability [6].

The Paper is organized as follows: Section 2 explores the related works in the area and Section 3 discusses the case study on Load and Performance Testing. Section 4 proposed a new tool for Navigability testing and Section 6 suggests future works possible in the area.

\section{RELATED WORK}

Conallen [1] proposed an UML Extension for mapping web pages while Ricca and Tonnela [2] stress the use of information derived from representation models for performing testing of Web Applications. Lee and Offutt [3] apply mutant analyses for data interaction validation through XML messages in their approach christened "Interaction
Mutation". Ruffo et. al. [4] describe a process for testing web applications but do not report their used measurements.

\section{LOAD AND PERFORMANCE TESTING: A CASE STUDY}

An e-commerce application was tested by simulating Simultaneous Access through Virtual Clients. The Virtual Clients were supplied by a Load Generator and the defined Test Script was prepared to verigy the application behavior when Virtual Clients simultaneously insert items into the shopping cart.

The tool used was the WebLOAD 8.1.0 Open Source Tool from RadView with the following configuration settings: Duration Test of 10 minutes and Virtual Clients increasing in Linear Progression from 30 to 60.

Some of the measures collected during the Load and Performance Testing include:

i) Load Size - Number of Virtual Clients running during the last reporting interval

ii) Receive Time - Time elapsed between receiving first and last byte

iii) Connection Time - Time it takes from the beginning of the HTTP request to the Connection establishment

iv) Failed Hits - Total number of times virtual clients made HTTP requests but did not receive the correct HTTP response

v) Failed Connections - Total number of times virtual clients tried to connect to the application but were unsuccessful

The results showed that the application behaved properly with 30 virtual clients but as the number of virtual clients approached 60 the application became unstable.

\section{NAVIGABILITY TESTING}

The second important category of NFRs for Web Applications is Navigability. Algorithms used by search engines consider the navigability in order to sort up the position of web applications. A research by Ribeiro [5] emphasizes the importance of an e-commerce application being ranked on the first position.

This paper proposes a navigability testing tool based on the specification models related by Ricca and Tonnela [2]. 
The Proposed tool takes as input an XML file that contains the following information: an identifier, 1 for the main page; a URL; and the Page Relevance - a value in the scale of 1 to 5. 1 meaning least relevant and 5 meaning the most relevant.

From the supplied XML the tool will build a navigability tree (Figure 1) and will output the following measures:

i) Unreachable Pages - Number of pages in server not reachable

ii) Not Found Pages - Pages returing Not Found (404) Error

iii) Reachable Pages from Main Page - Number of pages not reachable from the main page

iv) Closed Cycles - a cyclic sequence of steps leading back to the initial page

Such a tool will be of immense assistance to web engineers in their endeavor to engineer web pages with good navigability features.

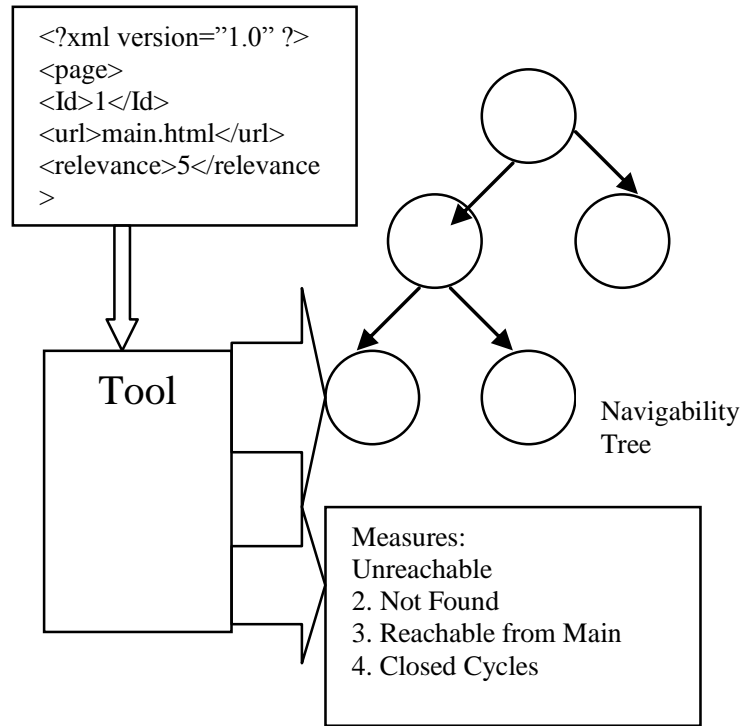

Figure 1 - The Proposed Tool

\section{CONCLUSION AND FUTURE WORKS}

NFRs for Web Applications are of paramount importance justifying their proper testing to ensure quality web applications. The paper attempted a case study on Load and Performance Testing using an open source tool and proposed a new tool for navigability testing.

The authors suggest the development of this tool and applying it on several web applications as a case study to explore its benefits.

\section{REFERENCES}

[1] J. Connalen, "Building Web Applications with UML", Addison-Wesley, Boston, MA, EUA, 2 ed., 2002.

[2] F. Ricca e P. Tonella, "Analysis and Testing of Web Applications" In: XXIII International Conference on Software Engineering - ICSE'01. p.25-34, Washington DC, EUA, May 2001 -IEEE Computer Society

[3] S. C Lee e J. Offutt, "Generating test cases for xml based web component interactions using mutation analysis" In: XII International Symposium on Software Reliability Engineering - ISSRE, p. 200, Washington DC, EUA, Nov 2001 IEEE Computer Society

[4] G. Ruffo, R. Schifanella, M. Sereno "A User Behavior Tailored Tool for Evaluating Web Application Performance" In: Network Computing and Applications - NCA 2004, p 77-86. Third IEEE International Symposium

[5] M. Ribeiro, http://www.midiadigital.com.br/index. php/2007/05/24/quanto-vale-o-primeiro-lugar-nogoogle/-visitada 19/06/2008

[6] Breno Lisi Romano et. al. "Software Testing for Web Applications Non-Functional requirements" 